\title{
The Influence of Creep on the Mechanical Properties of Calcium Carbonate Nanofiller Reinforced Polypropylene
}

\author{
Chrisopher Chukwutoo Ihueze ${ }^{1 *}$, Chinedum Ogonna Mgbemena ${ }^{2}$, Ugwu Sylveste $^{3}$ \\ ${ }^{1}$ Department of Industrial / Production Engineering, Nnamdi Azikiwe University Awka \\ ${ }^{2}$ Department of Mechanical Engineering, Nnamdi Azikiwe University Awka \\ ${ }^{3}$ Department of Mechanical Engineering, University of Nigeria \\ *Corresponding Author: ihuezechukwutoo@yahoo.com
}

\begin{abstract}
The study focused on experimental and classical data to establish some mechanical properties for optimum design of new polypropylene components to serve under creep environment. The creep studies recorded stress limits that never exceeded 24.19MPa and maximum creep modulus that never exceeded 1.49GPa as against the predictions of classical equations that gave 2.0GPa for PPCO and 2.46GPa for PPC2 at ambient conditions. The shear modulus and shear strength of the PPCO and the PPC2 are predicted as $0.75 \mathrm{GPa}$ and $120 \mathrm{MPa}$ respectively and $0.92 \mathrm{GPa}$ and $150 \mathrm{MPa}$ respectively while the yield strengths found to be about $13.19 \mathrm{MPa}$ and $13.20 \mathrm{MPa}$ respectively for PPCO and PPC2 at elastic strains 0.008 and 0.009 respectively. Further found are that as the material deforms the stiffness or modulus decrease, at low strains there is an elastic region, as temperature and applied stress increase the material becomes more flexible characterized with reduction in moduli. Plastic deformation at strains above 0.01 resulted to strain- hardening or strain-strengthening that manifested as the increasing area ratios and associated creep cold work. Also established by this study is a computational model for evaluating the elastic modulus of polypropylene matrix based material as expressed in equation (6). Both the Halphin-Tsai and the Birintrup equations for elastic modulus of unidirectional fibre composites were confirmed to be appropriate for prediction of elastic modulus of nanofiller composites with polymer matrix.
\end{abstract}

Keywords: Influence of creep, Mechanical properties, Calcium carbonate nanofiller, Reinforced Polypropylene. 


\section{INTRODUCTION}

Polymeric materials exhibit properties which come somewhere between elastic and viscous properties and are controlled by elastic and viscous constants called modulus and viscosity respectively making the mechanical properties of plastics to be viscoelastic [1]. This means that they vary with time under load, the rate of loading and the temperature and the creep limits of plastic composites need to be established because of involvement of plastics in most recent designs such as in multi-layer moldings, design of snap fits, design of ribbed sections and in design of light weight structures in everyday use.

Young's modulus is low for plastics and never constant compared with metals; resistance to deflection (stiffness) is often a concern regarding the use of plastics and the stiffness of a structure is dependent on the elastic modulus of the material and the part geometry.

Though many scholars such as [2-8] have worked extensively on the reinforcement of polypropylene with calcium carbonate nanofiller, studies are yet to advance on the limiting creep properties of polypropylene composites with calcium carbonate nanofiller. This study in order to address this pertinent issue used experimental and classical results to study the creep limiting properties of polypropylene and its calcium carbonate nanofiller composite.

Most mechanical properties are structure -sensitive and are therefore affected by changes in either the lattice structure or the microstructure. However modulus of elasticity is one property that is structure insensitive. The modulus of elasticity of material is the same regardless of grain size, amount of cold work, or microstructure while the ductility and toughness that are structure sensitive vary with the amount of cold work and/or grain size. When a crystalline material is plastically deformed, there is an avalanche of dislocations called slip that terminates at the grain boundaries, leading to mass movement of a body of atoms along a crystallographic plane [9].

\section{METHODOLOGY}

The methods of this study used the experimental tensile and tensile creep test results conducted on calcium carbonate nanofiller reinforced polypropylene composite by [10] with classical data and relations to evaluate the limiting properties of polypropylene as a new material.

\subsection{Use of Classical Relations of Composite Elastic Modulus}

The mass of a composite is the sum of the masses of the matrix (polymer) and the re-enforcing phase (filler). The properties of a composite material are then function of the starting materials [11] so that the following relations are found in literature for estimating the elastic modulus of 
particulate fillers [8]. The modulus of elasticity of the particle filled composite may be predicted using the following equations:

$E_{c}=E_{p} \varphi_{p}+E_{f} \varphi_{f}$

$E_{c}=\frac{E_{p} E_{f}}{E_{p} \varphi_{f}+E_{f} \varphi_{p}}$

$\mathrm{E}=\mathrm{E}_{\mathrm{m}}\left[1+2.5 \varphi+14.1 \varphi^{2}\right]$

where $\mathrm{E}=$ Modulus of elasticity, $\varphi=$ volume fractions, Subscripts $\mathrm{c}, \mathrm{f}$ and $\mathrm{p}$ represent the composites, filler and polymer.

\subsubsection{Estimation of elastic modulus of the composite}

The elastic modulus of the composite estimated with equations (1, 2 and 3$)$ in [10] is as presented in Table 1.

Table 1: Computed Composite Modulus with Existing Relations

\begin{tabular}{|l|l|l|l|l|}
\hline$\varphi_{\mathrm{p}}$ & $\varphi_{\mathrm{f}}$ & $\begin{array}{l}\text { Eq.(1), } \\
\text { E(GPa) }\end{array}$ & $\begin{array}{l}\text { Eq.(2), } \\
\text { E(GPa) }\end{array}$ & $\begin{array}{l}\text { Eq.(3), } \\
\text { E(GPa) }\end{array}$ \\
\hline 0.95 & 0.05 & 3.162 & 2.05500444 & 2.21191 \\
\hline 0.9 & 0.01 & 2.024 & 2.17595518 & 2.00928 \\
\hline 0.85 & 0.15 & 5.566 & 2.27560954 & 2.75718 \\
\hline 0.8 & 0.2 & 6.768 & 2.40468101 & 3.05054 \\
\hline 0.75 & 0.25 & 7.97 & 2.54927464 & 3.35773 \\
\hline 0.7 & 0.3 & 9.172 & 2.7123696 & 3.67872 \\
\hline 0.65 & 0.35 & 10.374 & 2.89775958 & 4.01354 \\
\hline 0.6 & 0.4 & 11.576 & 3.11035156 & 4.36218 \\
\hline 0.55 & 0.45 & 12.778 & 3.35660651 & 4.72463 \\
\hline 0.5 & 0.5 & 13.98 & 3.64520744 & 5.1009 \\
\hline 0.4 & 0.6 & 16.384 & 4.40221147 & 5.8949 \\
\hline
\end{tabular}

This study further employed the Halphin-Tsai and Brintrup equations for composite modulus expressed in equation (4 and 5) respectively [12] to come up with simpler and if possible better approximation for composite elastic modulus.

$\mathrm{E}=\mathrm{E}_{\mathrm{m}}\left(\frac{1+2 \beta \varphi}{1-\beta \varphi}\right)$

Where

$\beta=\frac{\left(E_{f} / E_{m}\right)-1}{\left(E_{f} / E_{m}\right)+2}$ 
$E=\frac{E^{m} E_{f}}{E_{f}(1-)+\varphi E^{m}}$

$\mathrm{E}^{\mathrm{m}}=\mathrm{E}_{\mathrm{m}} /\left(1-v^{2}{ }_{\mathrm{m}}\right)$

Where

$v_{\mathrm{m}}=$ Poisson ratio and for $\mathrm{PP}=0.34$.

The estimations of composite elastic modulus with equations (4 and 5) are presented in Table 2

Table 2: Composites elastic modulus with equations (4 and 5)

\begin{tabular}{|l|l|l|l|l|l|}
\hline $\begin{array}{l}\text { Specimen } \\
\text { code }\end{array}$ & $\mathrm{V}_{\mathrm{m}}$ & $\begin{array}{l}\mathrm{V}_{\mathrm{f}}= \\
\varphi\end{array}$ & $\mathrm{E}_{\mathrm{H}}$ & $\mathrm{E}_{\mathrm{B}}$ & $\begin{array}{l}\left(\mathrm{E}_{\mathrm{H}}+\mathrm{E}_{\mathrm{B}}\right) / 2 \\
=\mathrm{E}\end{array}$ \\
\hline PPCO & 1 & 0 & 1.96 & 2.22 & 2.09 \\
\hline PPC1 & 0.95 & 0.05 & 2.21 & 2.33 & 2.27 \\
\hline PPC2 & 0.9 & 0.1 & 2.47 & 2.44 & 2.46 \\
\hline PPC3 & 0.85 & 0.15 & 2.77 & 2.57 & 2.67 \\
\hline PPC4 & 0.8 & 0.2 & 3.09 & 2.72 & 2.91 \\
\hline PPC5 & 0.75 & 0.25 & 3.44 & 2.88 & 3.16 \\
\hline
\end{tabular}

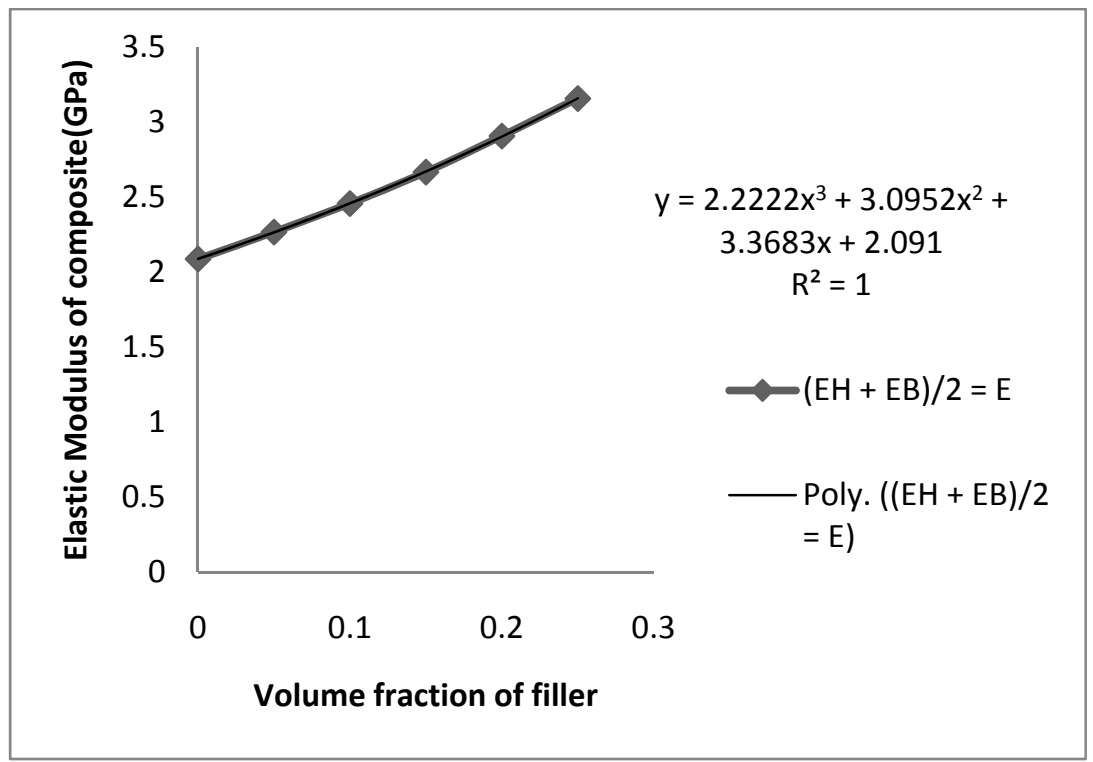

Figure 1: Elastic Modulus - Volume fraction of Filler

Through Figure 1 generated from predictions of Table 2 a cubic polynomial equation relating elastic modulus and volume fraction was established in this study as $\mathrm{E}=2.222 \varphi^{3}+3.095 \varphi^{2}+3.368 \varphi+2.091$ 


\subsection{Creep Testing and Computations}

TecQuipment creep equipment, model SM106 MKII was used to test PP and $\mathrm{PPCaCO}_{3}$ nanofiller composite of various volume fractions of $\mathrm{CaCo}_{3}$ nano filler at temperatures $25^{\mathrm{O}} \mathrm{C}$, $50^{\circ} \mathrm{C}$ and $70{ }^{\circ} \mathrm{C}$ respectively at various stresses to establish creep properties of the $\mathrm{PPCaCO}_{3}$ nanofiller composite [10] and the results presented in the following tables with the instantaneous cross sectional area of sample $A_{f}$, creep modulus $E(t)$ and creep compliance $C(t)$ evaluated according to the relations of $[9,12]$ expressed as follows

$\varepsilon=\ln \frac{A_{O}}{A_{f}}$

Where $\varepsilon$ is the true strain or natural strain expressed as

$\varepsilon=\ln (\mathrm{n}+1)$

Where $\mathrm{n}$ is the nominal strain or the engineering strain evaluated during the tensile test by measuring the percentage elongation of specimen

$\mathrm{E}(\mathrm{t})=\frac{\mathrm{s}}{\mathcal{\varepsilon}(\mathrm{t})}$

The creep modulus will vary with time, i.e decrease as time increases; sometimes creep compliance is used instead of creep modulus and is expressed as

$\mathrm{C}(\mathrm{t}) \quad=\frac{1}{\mathrm{E}(\mathrm{t})}=\frac{\varepsilon(\mathrm{t})}{\mathrm{s}}$

where $s$ is the constant creep stress and $\varepsilon(t)$ is the natural strain at time $t$. In this work $n(t)=\varepsilon(t)$ and $\sigma$ is the measured stress at experimental time $t$. The experimental creep results are presented in Tables 3-12.

Table 3: Experimental Creep Results obtained for PPC-0 at $13.08 \mathrm{MPa}, 25^{\circ} \mathrm{C}$ Ambient Condition

\begin{tabular}{|l|l|l|l|l|l|l|l|}
\hline $\mathrm{t}(\mathrm{hrs})$ & $\mathcal{E}(\mathrm{t})$ & $\mathrm{A}_{\mathrm{f}}$ & Arr & $\sigma(\mathrm{MPa})$ & $\mathrm{Ei}(\mathrm{GPa})$ & $\mathrm{E}(\mathrm{t})(\mathrm{MPa})$ & $\begin{array}{l}\mathrm{C}(\mathrm{t}) \\
\left(\mathrm{MPa}^{-1}\right)\end{array}$ \\
\hline 0.000 & 0.008 & 2381 & 1.0079798 & 13.19 & 1.6490 & 1.688 & 0.592 \\
\hline 0.277 & 0.010 & 2376.1 & 1.0100585 & 13.21 & 1.3210 & 1.350 & 0.741 \\
\hline 0.555 & 0.012 & 2371.4 & 1.0120604 & 13.24 & 1.1033 & 1.125 & 0.899 \\
\hline 0.833 & 0.015 & 2364.3 & 1.0150996 & 13.28 & 0.8853 & 0.900 & 1.111 \\
\hline 1.111 & 0.016 & 2361.9 & 1.0161311 & 13.29 & 0.8306 & 0.844 & 1.185 \\
\hline
\end{tabular}




\begin{tabular}{|l|l|l|l|l|l|l|l|}
1.388 & 0.017 & 2359.5 & 1.0171647 & 13.3 & 0.7824 & 0.794 & 1.259 \\
\hline 1.667 & 0.018 & 2357.2 & 1.0181571 & 13.32 & 0.7400 & 0.750 & 1.333 \\
\hline 1.944 & 0.019 & 2354.8 & 1.0191948 & 13.33 & 0.7016 & 0.711 & 1.406 \\
\hline 2.222 & 0.021 & 2350.1 & 1.0212331 & 13.36 & 0.6362 & 0.643 & 1.555 \\
\hline 2.500 & 0.022 & 2347.8 & 1.0222336 & 13.37 & 0.6077 & 0.614 & 1.629 \\
\hline 2.778 & 0.024 & 2343.1 & 1.0242841 & 13.4 & 0.5583 & 0.563 & 1.776 \\
\hline 3.056 & 0.028 & 2333.7 & 1.0284098 & 13.45 & 0.4804 & 0.482 & 2.075 \\
\hline 3.333 & 0.030 & 2329.1 & 1.0304409 & 13.48 & 0.4493 & 0.45 & 2.222 \\
\hline 3.611 & 0.031 & 2326.7 & 1.0315038 & 13.49 & 0.4352 & 0.435 & 2.299 \\
\hline 3.889 & 0.031 & 2326.7 & 1.0315038 & 13.49 & 0.4352 & 0.435 & 2.299 \\
\hline 4.167 & 0.032 & 2324.4 & 1.0325245 & 13.51 & 0.4222 & 0.422 & 2.37 \\
\hline 4.444 & 0.032 & 2324.4 & 1.0325245 & 13.51 & 0.4222 & 0.422 & 2.37 \\
\hline
\end{tabular}

Table 4: Experimental Creep Results obtained for PPC-2 at $13.08 \mathrm{MPa}, 25^{0} \mathrm{C}$ Ambient Condition

\begin{tabular}{|l|l|l|l|l|l|l|l|}
\hline$t(h r s)$ & $\varepsilon(t)$ & $A_{f}$ & Arr & $\sigma(\mathrm{MPa})$ & Ei(GPa) & $\mathrm{E}(\mathrm{t})(\mathrm{MPa})$ & $\begin{array}{l}\mathrm{C}(\mathrm{t}) \\
\left(\mathrm{MPa}^{-1}\right)\end{array}$ \\
\hline 0 & 0.009 & 2378.5 & 1.0090 & 13.2 & 1.4667 & 1.489 & 0.672 \\
\hline 0.277 & 0.012 & 2371.4 & 1.0120 & 13.24 & 1.1033 & 1.117 & 0.895 \\
\hline 0.555 & 0.013 & 2369 & 1.0130 & 13.25 & 1.0192 & 1.031 & 0.97 \\
\hline 0.833 & 0.015 & 2364.3 & 1.0151 & 13.28 & 0.8853 & 0.893 & 1.12 \\
\hline 1.111 & 0.017 & 2359.5 & 1.0171 & 13.3 & 0.7824 & 0.788 & 1.269 \\
\hline 1.388 & 0.018 & 2357.2 & 1.0181 & 13.32 & 0.74 & 0.744 & 1.3 \\
\hline 1.667 & 0.019 & 2354.8 & 1.0191 & 13.33 & 0.7016 & 0.705 & 1.425 \\
\hline 1.944 & 0.02 & 2352.5 & 1.0201 & 13.34 & 0.667 & 0.67 & 1.499 \\
\hline 2.222 & 0.021 & 2350.1 & 1.0212 & 13.36 & 0.6362 & 0.638 & 1.572 \\
\hline 2.5 & 0.023 & 2345.4 & 1.0232 & 13.38 & 0.5817 & 0.583 & 1.719 \\
\hline 2.778 & 0.024 & 2343.1 & 1.0242 & 13.4 & 0.5583 & 0.558 & 1.791 \\
\hline 3.056 & 0.025 & 2340.7 & 1.0253 & 13.41 & 0.5364 & 0.536 & 1.868 \\
\hline 3.333 & 0.026 & 2338.4 & 1.0263 & 13.42 & 0.5162 & 0.515 & 1.937 \\
\hline 3.611 & 0.028 & 2333.7 & 1.0283 & 13.45 & 0.4804 & 0.479 & 2.082 \\
\hline 3.889 & 0.029 & 2331.4 & 1.0294 & 13.46 & 0.4641 & 0.462 & 2.155 \\
\hline 4.167 & 0.03 & 2329.1 & 1.0304 & 13.48 & 0.4493 & 0.447 & 2.226 \\
\hline 4.444 & 0.03 & 2329.1 & 1.0304 & 13.48 & 0.4493 & 0.447 & 2.226 \\
\hline
\end{tabular}


Table 5: Experimental Creep Results obtained for PPC-0 at $19.60 \mathrm{MPa}, 25^{\circ} \mathrm{C}$ Ambient Condition.

\begin{tabular}{|l|l|l|l|l|l|l|l|}
\hline $\mathrm{t}(\mathrm{hrs})$ & $\varepsilon(\mathrm{t})$ & $\mathrm{A}_{\mathrm{f}}$ & Arr & $\sigma(\mathrm{MPa})$ & $\mathrm{Ei}(\mathrm{GPa})$ & $\mathrm{E}(\mathrm{t})(\mathrm{MPa})$ & $\begin{array}{l}\mathrm{C}(\mathrm{t}) \\
\left(\mathrm{MPa}^{-1}\right)\end{array}$ \\
\hline 0 & 0.015 & 2364.3 & 1.01511249 & 19.9 & 1.3264 & 1.353 & 0.739 \\
\hline 0.28 & 0.03 & 2329.1 & 1.03045422 & 20.2 & 0.6732 & 0.677 & 1.477 \\
\hline 0.56 & 0.033 & 2322.1 & 1.03355167 & 20.26 & 0.6139 & 0.615 & 1.626 \\
\hline 0.83 & 0.035 & 2317.5 & 1.03562105 & 20.3 & 0.58 & 0.58 & 1.724 \\
\hline 1.11 & 0.037 & 2312.8 & 1.03769424 & 20.34 & 0.5497 & 0.549 & 1.821 \\
\hline 1.39 & 0.04 & 2305.9 & 1.04081287 & 20.4 & 0.51 & 0.508 & 1.969 \\
\hline 1.67 & 0.042 & 2305.9 & 1.04081287 & 20.44 & 0.4867 & 0.483 & 2.07 \\
\hline 1.94 & 0.045 & 2301.3 & 1.04289333 & 20.5 & 0.4556 & 0.451 & 2.217 \\
\hline 2.22 & 0.048 & 2294.4 & 1.04602966 & 20.56 & 0.4283 & 0.423 & 2.364 \\
\hline 2.5 & 0.05 & 2283 & 1.05127138 & 20.6 & 0.4121 & 0.406 & 2.463 \\
\hline 2.78 & 0.053 & 2276.1 & 1.05443059 & 20.67 & 0.3899 & 0.383 & 2.611 \\
\hline 3.06 & 0.056 & 2269.3 & 1.05759951 & 20.73 & 0.3702 & 0.363 & 2.755 \\
\hline 3.33 & 0.058 & 2264.8 & 1.05971494 & 20.77 & 0.3581 & 0.35 & 2.857 \\
\hline 3.61 & 0.06 & 2260.2 & 1.06183414 & 20.81 & 0.3469 & 0.338 & 2.959 \\
\hline
\end{tabular}

Table 6: Experimental Creep Results obtained for PPC-2 at $19.60 \mathrm{MPa}, 25^{\circ} \mathrm{C}$ Ambient Condition.

\begin{tabular}{|l|l|l|l|l|l|l|l|}
\hline$t(h r s)$ & $\varepsilon(t)$ & $A_{f}$ & Arr & $\sigma(\mathrm{MPa})$ & $\mathrm{Ei}(\mathrm{GPa})$ & $\mathrm{E}(\mathrm{t})(\mathrm{MPa})$ & $\begin{array}{l}\mathrm{C}(\mathrm{t}) \\
\left(\mathrm{MPa}^{-1}\right)\end{array}$ \\
\hline 0 & 0.015 & 2364.3 & 1.01511 & 19.9 & 1.3264 & 1.353 & 0.739 \\
\hline 0.28 & 0.029 & 2331.4 & 1.02942 & 20.18 & 0.6958 & 0.7 & 1.429 \\
\hline 0.56 & 0.032 & 2324.4 & 1.03251 & 20.24 & 0.6324 & 0.634 & 1.577 \\
\hline 0.83 & 0.033 & 2322.1 & 1.03355 & 20.26 & 0.6139 & 0.615 & 1.626 \\
\hline 1.11 & 0.034 & 2319.8 & 1.0345 & 20.28 & 0.5964 & 0.597 & 1.675 \\
\hline 1.39 & 0.035 & 2317.5 & 1.03562 & 20.3 & 0.58 & 0.58 & 1.724 \\
\hline 1.67 & 0.036 & 2312.8 & 1.03769 & 20.34 & 0.565 & 0.563 & 1.776 \\
\hline 1.94 & 0.038 & 2310.5 & 1.03873 & 20.36 & 0.5358 & 0.534 & 1.873 \\
\hline 2.22 & 0.039 & 2308.2 & 1.03977 & 20.38 & 0.5226 & 0.521 & 1.919 \\
\hline 2.5 & 0.04 & 2305.9 & 1.04081 & 20.4 & 0.51 & 0.508 & 1.969 \\
\hline 2.78 & 0.042 & 2301.3 & 1.04289 & 20.44 & 0.4867 & 0.483 & 2.07 \\
\hline 3.06 & 0.044 & 2296.7 & 1.04498 & 20.48 & 0.4655 & 0.461 & 2.169 \\
\hline
\end{tabular}




\begin{tabular}{|l|l|l|l|l|l|l|l|}
3.33 & 0.046 & 2292.1 & 1.04707 & 20.52 & 0.4461 & 0.441 & 2.268 \\
\hline 3.61 & 0.05 & 2283 & 1.05127 & 20.6 & 0.4121 & 0.406 & 2.463 \\
\hline
\end{tabular}

Table 7: Experimental Creep Results obtained for PPC-0 at $22.87 \mathrm{MPa}, 25^{\circ} \mathrm{C}$ Ambient Condition

\begin{tabular}{|l|l|l|l|l|l|l|l|}
\hline $\mathrm{t}(\mathrm{hrs})$ & $\varepsilon(\mathrm{t})$ & $\mathrm{A}_{\mathrm{f}}$ & Arr & $\sigma(\mathrm{MPa})$ & $\mathrm{Ei}(\mathrm{GPa})$ & $\mathrm{E}(\mathrm{t})(\mathrm{MPa})$ & $\begin{array}{l}\mathrm{C}(\mathrm{t}) \\
\left(\mathrm{MPa}^{-1}\right)\end{array}$ \\
\hline 0 & 0.019 & 2354.8 & 1.01918185 & 23.31 & 1.2268 & 1.263 & 0.792 \\
\hline 0.056 & 0.02 & 2352.5 & 1.02019996 & 23.33 & 1.1167 & 1.2 & 0.833 \\
\hline 0.083 & 0.021 & 2350.1 & 1.0212201 & 23.36 & 1.1122 & 1.143 & 0.875 \\
\hline 0.139 & 0.03 & 2329.1 & 1.03045422 & 23.57 & 0.7856 & 0.8 & 1.25 \\
\hline 0.222 & 0.033 & 2322.1 & 1.03355167 & 23.64 & 0.7163 & 0.727 & 1.376 \\
\hline 0.278 & 0.04 & 2305.9 & 1.04081287 & 23.8 & 0.5951 & 0.6 & 1.667 \\
\hline 0.417 & 0.046 & 2296.7 & 1.04498213 & 23.9 & 0.5195 & 0.522 & 1.916 \\
\hline 0.556 & 0.05 & 2283 & 1.05127138 & 24.04 & 0.4809 & 0.48 & 2.083 \\
\hline 0.694 & 0.052 & 2278.4 & 1.05337541 & 24.09 & 0.4633 & 0.462 & 2.165 \\
\hline 0.833 & 0.056 & 2269.3 & 1.05759951 & 24.19 & 0.4319 & 0.429 & 2.331 \\
\hline
\end{tabular}

Table 8: Experimental Creep Results obtained for PPC-2 at $22.87 \mathrm{MPa}, 25^{\circ} \mathrm{C}$ Ambient Condition

\begin{tabular}{|l|l|l|l|l|l|l|l|}
\hline $\mathrm{t}(\mathrm{hrs})$ & $\varepsilon(\mathrm{t})$ & $\mathrm{A}_{\mathrm{f}}$ & Arr & $\sigma(\mathrm{MPa})$ & $\mathrm{Ei}(\mathrm{GPa})$ & $\mathrm{E}(\mathrm{t})(\mathrm{MPa})$ & $\begin{array}{l}\mathrm{C}(\mathrm{t}) \\
\left(\mathrm{MPa}^{-1}\right)\end{array}$ \\
\hline 0 & 0.019 & 2354.8 & 1.01918185 & 23.31 & 1.2268 & 1.263 & 0.792 \\
\hline 0.056 & 0.02 & 2352.5 & 1.02019996 & 23.33 & 1.1167 & 1.2 & 0.833 \\
\hline 0.083 & 0.021 & 2350.1 & 1.0212201 & 23.36 & 1.1122 & 1.091 & 0.917 \\
\hline 0.139 & 0.03 & 2329.1 & 1.03045422 & 23.57 & 0.7856 & 0.615 & 1.626 \\
\hline 0.222 & 0.033 & 2322.1 & 1.03355167 & 23.64 & 0.7163 & 0.571 & 1.751 \\
\hline 0.278 & 0.04 & 2305.9 & 1.04081287 & 23.8 & 0.5951 & 0.522 & 1.916 \\
\hline 0.417 & 0.046 & 2296.7 & 1.04498213 & 23.9 & 0.5195 & 0.5 & 2 \\
\hline 0.556 & 0.05 & 2283 & 1.05127138 & 24.04 & 0.4809 & 0.48 & 2.083 \\
\hline 0.694 & 0.052 & 2278.4 & 1.05337541 & 24.09 & 0.4633 & 0.462 & 2.165 \\
\hline 0.833 & 0.056 & 2269.3 & 1.05759951 & 24.19 & 0.4319 & 0.429 & 2.331 \\
\hline
\end{tabular}


Table 9: Experimental Creep Results obtained for PPC-0 at $50^{\circ} \mathrm{C}$, and Stress of $13.08 \mathrm{MPa}$

\begin{tabular}{|l|l|l|l|l|l|l|l|}
\hline$t(h r s)$ & $\varepsilon(t)$ & $A_{f}$ & Arr & $\sigma(\mathrm{MPa})$ & Ei(GPa) & $\mathrm{E}(\mathrm{t})(\mathrm{MPa})$ & $\begin{array}{l}\mathrm{C}(\mathrm{t}) \\
\left(\mathrm{MPa}^{-1}\right)\end{array}$ \\
\hline 0.083 & 0.02 & 2352.5 & 1.0202043 & 13.34 & 0.667 & 0.695 & 1.439 \\
\hline 0.167 & 0.022 & 2347.9 & 1.02219875 & 13.37 & 0.608 & 0.632 & 1.582 \\
\hline 0.25 & 0.03 & 2329 & 1.03049846 & 13.48 & 0.449 & 0.463 & 2.16 \\
\hline 0.333 & 0.034 & 2319.8 & 1.03458087 & 13.53 & 0.398 & 0.409 & 2.444 \\
\hline 0.417 & 0.04 & 2305.9 & 1.04080836 & 13.61 & 0.34 & 0.348 & 2.874 \\
\hline 0.5 & 0.046 & 2292.1 & 1.04707017 & 13.7 & 0.298 & 0.302 & 3.311 \\
\hline 0.583 & 0.05 & 2283 & 1.05127138 & 13.75 & 0.275 & 0.278 & 3.597 \\
\hline 0.667 & 0.058 & 2264.8 & 1.05971026 & 13.86 & 0.239 & 0.24 & 4.167 \\
\hline 0.75 & 0.058 & 2264.8 & 1.05971026 & 13.86 & 0.239 & 0.24 & 4.167 \\
\hline 0.833 & 0.058 & 2264.8 & 1.05971026 & 13.86 & 0.239 & 0.24 & 4.167 \\
\hline
\end{tabular}

Table 10: Experimental Creep Results obtained for PPC-2 at $50^{\circ} \mathrm{C}$, and Stress of 13.08MPa

\begin{tabular}{|l|l|l|l|l|l|l|l|}
\hline$t(h r s)$ & $\varepsilon(t)$ & $A_{f}$ & Arr & $\sigma(\mathrm{MPa})$ & Ei(GPa) & E(t)(MPa) & $\begin{array}{l}\mathrm{C}(\mathrm{t}) \\
\left(\mathrm{MPa}^{-1}\right)\end{array}$ \\
\hline 0.083 & 0.018 & 2357.2 & 1.01816145 & 13.32 & 0.74 & 0.767 & 1.304 \\
\hline 0.167 & 0.022 & 2347.9 & 1.02219875 & 13.37 & 0.608 & 0.627 & 1.595 \\
\hline 0.25 & 0.026 & 2338.4 & 1.0263428 & 13.42 & 0.516 & 0.531 & 1.883 \\
\hline 0.333 & 0.03 & 2329 & 1.03049846 & 13.48 & 0.449 & 0.46 & 2.174 \\
\hline 0.417 & 0.033 & 2322.1 & 1.03355167 & 13.52 & 0.41 & 0.418 & 2.392 \\
\hline 0.5 & 0.04 & 2305.9 & 1.04080836 & 13.61 & 0.34 & 0.345 & 2.899 \\
\hline 0.583 & 0.046 & 2292.1 & 1.04707017 & 13.7 & 0.298 & 0.3 & 3.333 \\
\hline 0.667 & 0.05 & 2283 & 1.05127138 & 13.75 & 0.275 & 0.276 & 3.623 \\
\hline 0.75 & 0.054 & 2273.8 & 1.05548324 & 13.81 & 0.256 & 0.256 & 3.906 \\
\hline 0.833 & 0.058 & 2264.8 & 1.05971494 & 13.86 & 0.239 & 0.238 & 4.202 \\
\hline
\end{tabular}

Table 11: Experimental Creep Results obtained for PPC-0 at $70^{\circ} \mathrm{C}$, and Stress of 13.08MPa

\begin{tabular}{|l|l|l|l|l|l|l|l|}
\hline $\mathrm{t}(\mathrm{hrs})$ & $\varepsilon(\mathrm{t})$ & $\mathrm{A}_{\mathrm{f}}$ & Arr & $\sigma(\mathrm{MPa})$ & $\mathrm{Ei}(\mathrm{GPa})$ & $\mathrm{E}(\mathrm{t})(\mathrm{MPa})$ & $\begin{array}{l}\mathrm{C}(\mathrm{t}) \\
\left(\mathrm{MPa}^{-1}\right)\end{array}$ \\
\hline 0.083 & 0.019 & 2354.8 & 1.01919916 & 13.33 & 0.702 & 0.737 & 1.357 \\
\hline 0.167 & 0.025 & 2340.7 & 1.02532116 & 13.41 & 0.536 & 0.56 & 1.786 \\
\hline
\end{tabular}




\begin{tabular}{|l|l|l|l|l|l|l|l|}
0.25 & 0.038 & 2310.6 & 1.03870024 & 13.59 & 0.358 & 0.368 & 2.717 \\
\hline 0.333 & 0.05 & 2283 & 1.05127138 & 13.75 & 0.275 & 0.28 & 3.571 \\
\hline 0.417 & 0.056 & 2269.3 & 1.05759951 & 13.83 & 0.247 & 0.25 & 4 \\
\hline 0.5 & 0.063 & 2253.5 & 1.06502889 & 13.93 & 0.221 & 0.222 & 4.505 \\
\hline 0.583 & 0.069 & 2240 & 1.07143814 & 14.01 & 0.203 & 0.203 & 4.926 \\
\hline 0.667 & 0.075 & 2226.6 & 1.07788627 & 14.01 & 0.188 & 0.187 & 5.348 \\
\hline
\end{tabular}

Table 12: Experimental Creep Results obtained for PPC-2 at $70^{\circ} \mathrm{C}$, and Stress of 13.08MPa

\begin{tabular}{|l|l|l|l|l|l|l|l|}
\hline$t(h r s)$ & $\varepsilon(t)$ & $A_{f}$ & Arr & $\sigma(\mathrm{MPa})$ & $\mathrm{Ei}(\mathrm{GPa})$ & $\mathrm{E}(\mathrm{t})(\mathrm{MPa})$ & $\begin{array}{l}\mathrm{C}(\mathrm{t}) \\
\left(\mathrm{MPa}^{-1}\right)\end{array}$ \\
\hline 0.083 & 0.005 & 2388 & 1.0050125 & 13.15 & 2.63 & 2.68 & 0.373 \\
\hline 0.167 & 0.01 & 2376.1 & 1.01005 & 13.21 & 1.321 & 1.34 & 0.746 \\
\hline 0.25 & 0.015 & 2364.5 & 1.01501374 & 13.28 & 0.885 & 0.893 & 1.12 \\
\hline 0.333 & 0.02 & 2352.5 & 1.02019996 & 13.34 & 0.667 & 0.67 & 1.493 \\
\hline 0.417 & 0.023 & 2345.4 & 1.02327089 & 13.38 & 0.582 & 0.583 & 1.715 \\
\hline 0.5 & 0.025 & 2340.7 & 1.02532116 & 13.41 & 0.536 & 0.536 & 1.866 \\
\hline 0.583 & 0.03 & 2329.1 & 1.03045422 & 13.48 & 0.449 & 0.447 & 2.237 \\
\hline 0.667 & 0.035 & 2317.5 & 1.03562105 & 13.55 & 0.387 & 0.383 & 2.611 \\
\hline
\end{tabular}

\subsubsection{Estimation of amount of cold work}

The amount of cold work is defined as the percentage of reduction of cross-sectional area that is given the material by a plastic deformation process and is expressed mathematically as

$\mathrm{W}=\frac{\mathrm{A}_{0}-\mathrm{A}_{\mathrm{f}}}{\mathrm{A}_{0}}(100)=\left(1-\frac{A_{f}}{A_{0}}\right)(100)=\left(1-\frac{1}{A r r}\right)(100)$

The area ratios of all the operations are presented in Tables 3-12 as summarized in Table 13. Equation (10) is then employed with excel tools to compute the amount of cold work as presented in table 14a and $\mathrm{b}$, where the symbols Arr3-Arr12 represented the area ratios associated with Tables 3-12 and W3-W12 represented the cold work associated.

Table 13: Depiction of Area Ratios of all Creep Conditions

\begin{tabular}{|l|l|l|l|l|l|l|l|l|l|}
\hline Arr3 & Arr4 & Arr5 & Arr6 & Arr7 & Arr8 & Arr9 & Arr10 & Arr11 & Arr12 \\
\hline 1.008 & 1.009 & 1.0151 & 1.0151 & 1.0192 & 1.0192 & 1.0202 & 1.0182 & 1.0192 & 1.005 \\
\hline
\end{tabular}




\begin{tabular}{|l|l|l|l|l|l|l|l|l|l|}
1.0101 & 1.012 & 1.0305 & 1.0294 & 1.0202 & 1.0202 & 1.0222 & 1.0222 & 1.0253 & 1.0101 \\
\hline 1.0121 & 1.013 & 1.0336 & 1.0325 & 1.0212 & 1.0212 & 1.0305 & 1.0263 & 1.0387 & 1.015 \\
\hline 1.0151 & 1.0151 & 1.0356 & 1.0336 & 1.0305 & 1.0305 & 1.0346 & 1.0305 & 1.0513 & 1.0202 \\
\hline 1.0161 & 1.0171 & 1.0377 & 1.0345 & 1.0336 & 1.0336 & 1.0408 & 1.0336 & 1.0576 & 1.0233 \\
\hline 1.0172 & 1.0181 & 1.0408 & 1.0356 & 1.0408 & 1.0408 & 1.0471 & 1.0408 & 1.065 & 1.0253 \\
\hline 1.0182 & 1.0191 & 1.0408 & 1.0377 & 1.045 & 1.045 & 1.0513 & 1.0471 & 1.0714 & 1.0305 \\
\hline 1.0192 & 1.0201 & 1.0429 & 1.0387 & 1.0513 & 1.0513 & 1.0597 & 1.0513 & 1.0779 & 1.0356 \\
\hline 1.0212 & 1.0212 & 1.046 & 1.0398 & 1.0534 & 1.0534 & 1.0597 & 1.0555 & & \\
\hline 1.0222 & 1.0232 & 1.0513 & 1.0408 & 1.0576 & 1.0576 & 1.0597 & 1.0597 & & \\
\hline 1.0243 & 1.0242 & 1.0544 & 1.0429 & & & & & & \\
\hline 1.0284 & 1.0253 & 1.0576 & 1.045 & & & & & & \\
\hline 1.0304 & 1.0263 & 1.0597 & 1.0471 & & & & & & \\
\hline 1.0315 & 1.0283 & 1.0618 & 1.0513 & & & & & & \\
\hline 1.0315 & 1.0294 & & & & & & & & \\
\hline 1.0325 & 1.0304 & & & & & & & & \\
\hline 1.0325 & 1.0304 & & & & & & & & \\
\hline
\end{tabular}

Table 14a: Cold Work Results of Operations

\begin{tabular}{|l|l|l|l|l|l|l|r|r|r|}
\hline Arr3 & Arr4 & Arr5 & Arr6 & Arr7 & W3 & W4 & \multicolumn{1}{l|}{ W5 } & W6 & \multicolumn{1}{l|}{ W7 } \\
\hline 1.008 & 1.009 & 1.0151 & 1.0151 & 1.0192 & 0.791663 & 0.891972 & 1.48875 & 1.488509 & 1.882083 \\
\hline 1.0101 & 1.012 & 1.0305 & 1.0294 & 1.0202 & 0.995833 & 1.185771 & 2.955417 & 2.85792 & 1.98 \\
\hline 1.0121 & 1.013 & 1.0336 & 1.0325 & 1.0212 & 1.191668 & 1.283317 & 3.24625 & 3.148638 & 2.077916 \\
\hline 1.0151 & 1.0151 & 1.0356 & 1.0336 & 1.0305 & 1.487499 & 1.487538 & 3.439583 & 3.246094 & 2.955417 \\
\hline 1.0161 & 1.0171 & 1.0377 & 1.0345 & 1.0336 & 1.587502 & 1.681251 & 3.6325 & 3.334944 & 3.24625 \\
\hline 1.0172 & 1.0181 & 1.0408 & 1.0356 & 1.0408 & 1.687504 & 1.777821 & 3.92125 & 3.439486 & 3.92125 \\
\hline 1.0182 & 1.0191 & 1.0408 & 1.0377 & 1.045 & 1.78333 & 1.874203 & 3.92125 & 3.632106 & 4.304584 \\
\hline 1.0192 & 1.0201 & 1.0429 & 1.0387 & 1.0513 & 1.88333 & 1.970395 & 4.112916 & 3.728592 & 4.877083 \\
\hline 1.0212 & 1.0212 & 1.046 & 1.0398 & 1.0534 & 2.079163 & 2.075989 & 4.400416 & 3.824884 & 5.067083 \\
\hline 1.0222 & 1.0232 & 1.0513 & 1.0408 & 1.0576 & 2.175002 & 2.267396 & 4.877083 & 3.920985 & 5.44625 \\
\hline 1.0243 & 1.0242 & 1.0544 & 1.0429 & & 2.370836 & 2.36282 & 5.162084 & 4.11261 & \\
\hline 1.0284 & 1.0253 & 1.0576 & 1.045 & & 2.762498 & 2.46757 & 5.44625 & 4.304389 & \\
\hline 1.0304 & 1.0263 & 1.0597 & 1.0471 & & 2.954163 & 2.562604 & 5.635 & 4.495401 & \\
\hline 1.0315 & 1.0283 & 1.0618 & 1.0513 & & 3.054162 & 2.752115 & 5.823333 & 4.876958 & \\
\hline 1.0315 & 1.0294 & & & & 3.054162 & 2.856033 & & & \\
\hline 1.0325 & 1.0304 & & & & 3.149998 & 2.950311 & & & \\
\hline 1.0325 & 1.0304 & & & & 3.149998 & 2.950311 & & & \\
\hline
\end{tabular}


Table 14b: Cold Work Results of Operations

\begin{tabular}{|l|l|l|l|l|r|r|r|r|r|}
\hline Arr8 & Arr9 & Arr10 & Arr11 & ARR12 & W8 & W9 & W10 & W11 & \multicolumn{1}{l|}{ W12 } \\
\hline 1.0192 & 1.0202 & 1.0182 & 1.0192 & 1.005 & 1.882083 & 1.980417 & 1.78375 & 1.88375 & 0.49875 \\
\hline 1.0202 & 1.0222 & 1.0222 & 1.0253 & 1.0101 & 1.98 & 2.171667 & 2.171667 & 2.469583 & 0.995 \\
\hline 1.0212 & 1.0305 & 1.0263 & 1.0387 & 1.015 & 2.077916 & 2.959583 & 2.566667 & 3.725833 & 1.479166 \\
\hline 1.0305 & 1.0346 & 1.0305 & 1.0513 & 1.0202 & 2.955417 & 3.3425 & 2.959583 & 4.877083 & 1.98 \\
\hline 1.0336 & 1.0408 & 1.0336 & 1.0576 & 1.0233 & 3.24625 & 3.920833 & 3.24625 & 5.44625 & 2.274167 \\
\hline 1.0408 & 1.0471 & 1.0408 & 1.065 & 1.0253 & 3.92125 & 4.495417 & 3.920833 & 6.105833 & 2.469583 \\
\hline 1.045 & 1.0513 & 1.0471 & 1.0714 & 1.0305 & 4.304584 & 4.877083 & 4.495417 & 6.6675 & 2.955417 \\
\hline 1.0513 & 1.0597 & 1.0513 & 1.0779 & 1.0356 & 4.877083 & 5.634584 & 4.877083 & 7.225834 & 3.439583 \\
\hline 1.0534 & 1.0597 & 1.0555 & & & 5.067083 & 5.634584 & 5.256667 & & \\
\hline 1.0576 & 1.0597 & 1.0597 & & & 5.44625 & 5.634584 & 5.635 & & \\
\hline
\end{tabular}

\subsubsection{Limit stress-cold work for PPC0 and PPC2}

The influence of cold work on the strength property is shown on Table $15 \mathrm{a}$ and $\mathrm{b}$.

Table 15a: Cold Work Results of Operations

\begin{tabular}{|l|l|l|l|l|l|l|r|r|r|}
\hline$\sigma 3$ & $\sigma 4$ & $\sigma 5$ & $\sigma 6$ & $\sigma 7$ & $\mathrm{~W} 3$ & $\mathrm{~W} 4$ & $\mathrm{~W} 5$ & W6 & W7 \\
\hline 13.19 & 13.2 & 19.9 & 19.9 & 23.31 & 0.791663 & 0.891972 & 1.48875 & 1.488509 & 1.882083 \\
\hline 13.21 & 13.24 & 20.2 & 20.18 & 23.33 & 0.995833 & 1.185771 & 2.955417 & 2.85792 & 1.98 \\
\hline 13.24 & 13.25 & 20.26 & 20.24 & 23.36 & 1.191668 & 1.283317 & 3.24625 & 3.148638 & 2.077916 \\
\hline 13.28 & 13.28 & 20.3 & 20.26 & 23.57 & 1.487499 & 1.487538 & 3.439583 & 3.246094 & 2.955417 \\
\hline 13.29 & 13.3 & 20.34 & 20.28 & 23.64 & 1.587502 & 1.681251 & 3.6325 & 3.334944 & 3.24625 \\
\hline 13.3 & 13.32 & 20.4 & 20.3 & 23.8 & 1.687504 & 1.777821 & 3.92125 & 3.439486 & 3.92125 \\
\hline 13.32 & 13.33 & 20.44 & 20.34 & 23.9 & 1.78333 & 1.874203 & 3.92125 & 3.632106 & 4.304584 \\
\hline 13.33 & 13.34 & 20.5 & 20.36 & 24.04 & 1.88333 & 1.970395 & 4.112916 & 3.728592 & 4.877083 \\
\hline 13.36 & 13.36 & 20.56 & 20.38 & 24.09 & 2.079163 & 2.075989 & 4.400416 & 3.824884 & 5.067083 \\
\hline 13.37 & 13.38 & 20.6 & 20.4 & 24.19 & 2.175002 & 2.267396 & 4.877083 & 3.920985 & 5.44625 \\
\hline 13.4 & 13.4 & 20.67 & 20.44 & & 2.370836 & 2.36282 & 5.162084 & 4.11261 & \\
\hline 13.45 & 13.41 & 20.73 & 20.48 & & 2.762498 & 2.46757 & 5.44625 & 4.304389 & \\
\hline 13.48 & 13.42 & 20.77 & 20.52 & & 2.954163 & 2.562604 & 5.635 & 4.495401 & \\
\hline 13.49 & 13.45 & 20.81 & 20.6 & & 3.054162 & 2.752115 & 5.823333 & 4.876958 & \\
\hline 13.49 & 13.46 & & & & 3.054162 & 2.856033 & & & \\
\hline 13.51 & 13.48 & & & & 3.149998 & 2.950311 & & & \\
\hline 13.51 & 13.48 & & & & 3.149998 & 2.950311 & & & \\
\hline
\end{tabular}


Table 15b: Cold Work Results of Operations

\begin{tabular}{|l|l|l|l|l|r|r|r|r|r|}
\hline$\sigma 8$ & $\sigma 9$ & $\sigma 10$ & $\sigma 11$ & $\sigma 12$ & W8 & W9 & W10 & W11 & W12 \\
\hline 23.31 & 13.34 & 13.32 & 13.33 & 13.15 & 1.882083 & 1.980417 & 1.78375 & 1.88375 & 0.49875 \\
\hline 23.33 & 13.37 & 13.37 & 13.41 & 13.21 & 1.98 & 2.171667 & 2.171667 & 2.469583 & 0.995 \\
\hline 23.36 & 13.48 & 13.42 & 13.59 & 13.28 & 2.077916 & 2.959583 & 2.566667 & 3.725833 & 1.479166 \\
\hline 23.57 & 13.53 & 13.48 & 13.75 & 13.34 & 2.955417 & 3.3425 & 2.959583 & 4.877083 & 1.98 \\
\hline 23.64 & 13.61 & 13.52 & 13.83 & 13.38 & 3.24625 & 3.920833 & 3.24625 & 5.44625 & 2.274167 \\
\hline 23.8 & 13.7 & 13.61 & 13.93 & 13.41 & 3.92125 & 4.495417 & 3.920833 & 6.105833 & 2.469583 \\
\hline 23.9 & 13.75 & 13.7 & 14.01 & 13.48 & 4.304584 & 4.877083 & 4.495417 & 6.6675 & 2.955417 \\
\hline 24.04 & 13.86 & 13.75 & 14.01 & 13.55 & 4.877083 & 5.634584 & 4.877083 & 7.225834 & 3.439583 \\
\hline 24.09 & 13.86 & 13.81 & & & 5.067083 & 5.634584 & 5.256667 & & \\
\hline 24.19 & 13.86 & 13.86 & & & 5.44625 & 5.634584 & 5.635 & & \\
\hline
\end{tabular}

\section{ESTIMATION OF SLIP IN POLYPROPYLENE MATERIALS}

Slip will occur in polypropylene component when the yield strength is exceeded. The yield strength of polypropylene is in the range 12-43MPa [13]. Tables 3-12 showing creep stresses indicate the occurrence of lip due to low yield strength associated with creep. The shear strength of material is estimated with the classical relation

$\tau=\frac{G}{2 \pi}$

where $\mathrm{G}$ is the shear modulus estimated with the relation

$\mathrm{G}=\frac{\mathrm{E}}{2(1+v)}$

So that by using the values $\mathrm{E}=2 \mathrm{GPa}$ and $v=0.34$ the shear modulus and shear strength is evaluated for PPCO as $750 \mathrm{MPa}$ and $120 \mathrm{MPa}$ respectively and for PPC2 are $920 \mathrm{MPa}$ and $150 \mathrm{MPa}$ respectively.

\section{DISCUSSION OF RESULTS}

Table 1 and 2 show that the new PP (PPC2) has elastic modulus of 2-2.46GPa at optimum volume fraction of $0.10(10 \%)$ while table 2 distinctively show that neat $\mathrm{PP}(\mathrm{PPC} 0)$ has elastic modulus of about $2 \mathrm{GPa}$ at optimum volume fraction of 0.10 .

Table 3 and 4 at $13.08 \mathrm{MPa}$ applied static stress and ambient condition $25^{\circ} \mathrm{C}$ show the presence of primary creep stage, creep limit $13.51 \mathrm{MPa}$, elastic modulus $1.35 \mathrm{GPa}$ at 0.01 natural strain, modulus at fracture $0.422 \mathrm{GPa}$ and fracture strain of 0.032 for neat PP (PPC0) and for PPC2 show 
the presence of primary creep stage, creep limit $13.48 \mathrm{MPa}$, elastic modulus $1.49 \mathrm{GPa}$ at 0.01 natural strain, modulus at fracture $0.447 \mathrm{GPa}$ and fracture strain of 0.03 .

Table 5 and 6 at $19.60 \mathrm{MPa}$ applied static stress and ambient condition $25^{\circ} \mathrm{C}$ show the presence of primary creep stage with elastic strain 0.015 and modulus $1.353 \mathrm{GPa}$, creep limit $20.81 \mathrm{MPa}$, modulus at fracture $0.3338 \mathrm{GPa}$ and fracture strain of 0.06 for neat PP (PPC0) and for PPC 2 show the presence of primary creep stage with elastic strain 0.015 and modulus $1.353 \mathrm{GPa}$, creep limit 20.06 MPa, modulus at fracture $0.406 \mathrm{GPa}$ and fracture strain of 0.05 .

Table 7 and 8 at $22.88 \mathrm{MPa}$ applied static stress and ambient condition $25^{\circ} \mathrm{C}$ show the presence of primary creep stage with elastic strain 0.015 and modulus $1.263 \mathrm{GPa}$, creep limit $24.19 \mathrm{MPa}$, modulus at fracture $0.429 \mathrm{GPa}$ and fracture strain of 0.056 for neat PP (PPC0) and for PPC2 show the presence of primary creep stage with elastic strain 0.019 and modulus $1.263 \mathrm{GPa}$, creep limit 24.19MPa, modulus at fracture $0.429 \mathrm{GPa}$ and fracture strain of 0.056 also.

Table 9 and 10 at $13.08 \mathrm{MPa}$ applied static stress and ambient condition $50^{\circ} \mathrm{C}$ show the absence of primary creep stage and presence of creep limit $13.86 \mathrm{MPa}$, modulus at fracture $0.24 \mathrm{GPa}$ and fracture strain of 0.06 for neat PP (PPC0) and for PPC2 show the absence of primary creep stage and presence of creep limit $13.86 \mathrm{MPa}$, modulus at fracture $0.24 \mathrm{GPa}$ and fracture strain of 0.06 also.

Table 11 and 12 at $13.08 \mathrm{MPa}$ applied static stress and ambient condition $70^{\circ} \mathrm{C}$ show the absence of primary creep stage and presence of creep limit $14.01 \mathrm{MPa}$, modulus at fracture $0.187 \mathrm{GPa}$ and fracture strain of 0.08 for neat PP (PPC0) and for PPC2 show the absence of primary creep stage and presence of creep limit $13.55 \mathrm{MPa}$, modulus at fracture $0.383 \mathrm{GPa}$ and fracture strain of 0.035 The tensile strength of polypropylene is in the range $19.7-80 \mathrm{MPa}$ [12] by classical report and by experimental results of our previous report the tensile strength is $123 \mathrm{MPa}$ [10]. For the new material PPC2 our previous report gave the value of tensile strength as $45 \mathrm{MPa}$ [10]. From tables 3-12 the values of the recorded stress limits never exceeded 24.19MPa which is below the tensile limit obtained from classical reports showing the reducing influence of creep on the strength properties of PP. Further still on tables 3-12, notice that the maximum estimated elastic creep modulus at $1 \%$ natural strain approximately never exceeded $1.49 \mathrm{GPa}$ as against the predictions of classical equations that gave 2.0GPa for PPCO and 2.46GPa for PPC2. Creep therefore reduces the strength and stiffness properties of polypropylene and its nanofiller composites. Tables 3-12 clearly show that as the material deforms the stiffness or modulus decrease, at low strains there is an elastic region, as temperature and applied stress increase the material becomes more flexible characterized with reduction in moduli.

Plastic deformation at strains above 0.01 resulted to strain- hardening or stain-strengthening that manifested as the increasing area ratios and associated creep cold work as found in tables 3-12. 
The stress-strain plots of tables 3-12 are linear graphs giving the strain-strengthening equation of plastic deformation when plotted on logarithmic graph [9] as

$\sigma=c \varepsilon^{\mathrm{m}}$

$m$ is called the strain-strengthening exponent showing that strength increases with plastic strain operation increases. Figures 2 and 3 also show that the creep limit increases with increasing amount of cold work.
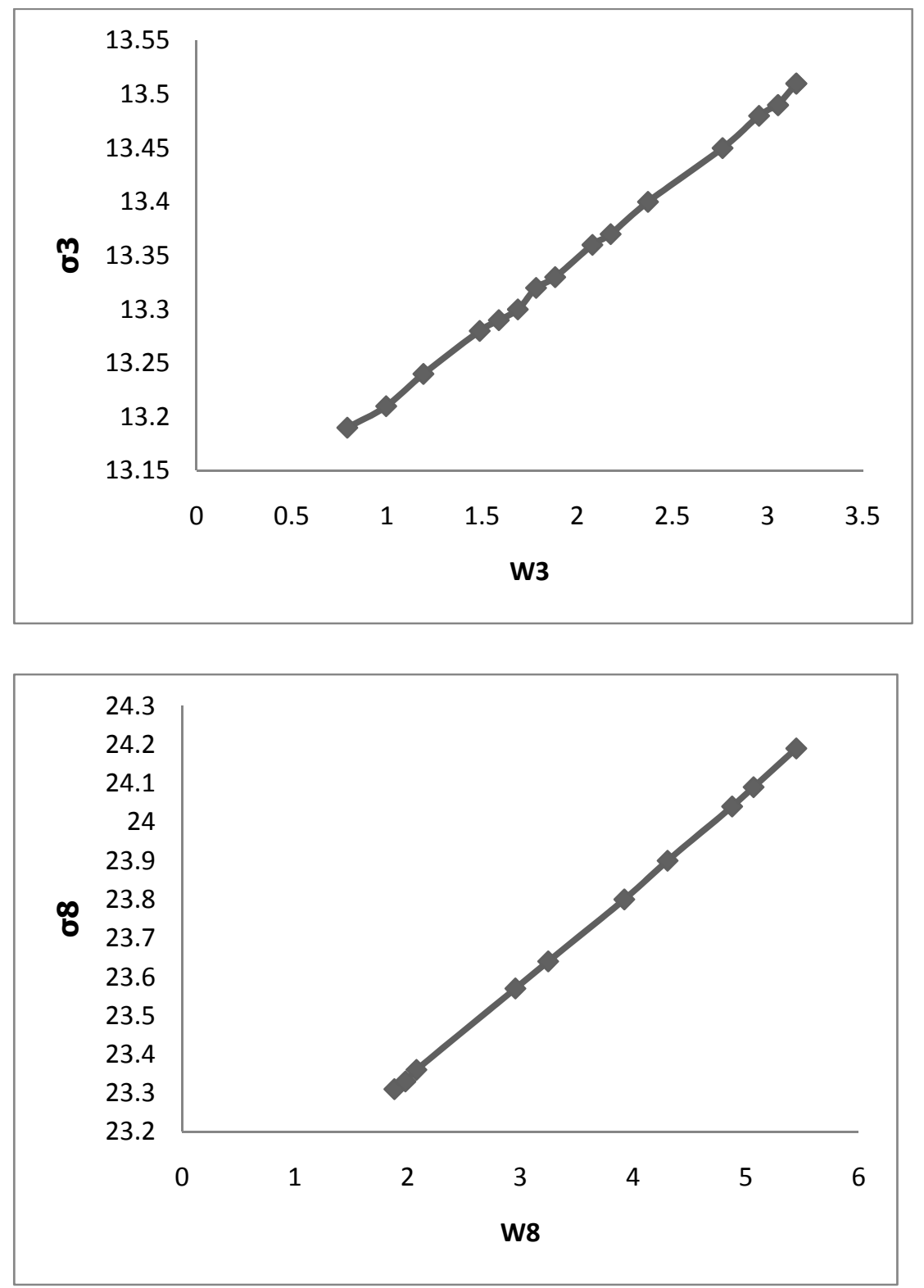


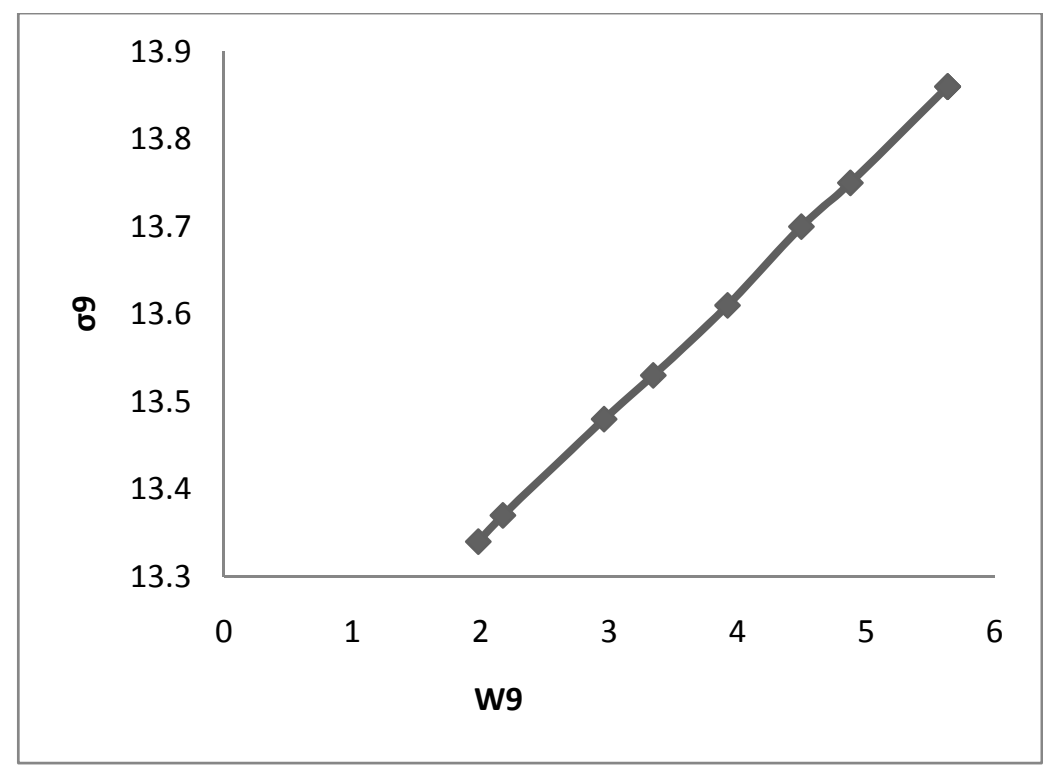

\section{Figure 2a, b, and c: Depiction showing stress-cold work relationship in creep analysis}

Table 3 and 4 established the shear strength of PPCO and PPC2 13.19MPa and 13.20MPa respectively at elastic strains of 0.008 and 0.009 while their shear moduli were estimated with equations $(11,12)$ as $0.75 \mathrm{GPa}$ and $0.92 \mathrm{GPa}$ respectively while their shear strengths were $120 \mathrm{MPa}$ and $150 \mathrm{MPa}$. These materials are then seen to be stronger in shear than in tension as the yield strength of this material under creep is about $13 \mathrm{MPa}$ compared to the classical range of 12$43 \mathrm{MPa}$ ) for this material[13]. The creep failure of these materials is therefore due to slip owing to mass movement of body of atoms that may form slip jog within the crystallographic plane since the yield strength of these materials was exceeded.

\section{CONCLUSION}

This study established the mechanical properties of polypropylene and that reinforced with calcium carbonate nanofiller as a new material under various serving creep conditions. Also established was that creep process may be a strengthening process slip occurring when the material yield strength is exceeded causes creep failure of polypropylene matrix composites. Plastic deformation at strains above 0.01 resulted to strain- hardening or strain-strengthening that manifested as the increased area ratios and associated creep cold work.

Also established by this study is a computational model for evaluating the elastic modulus of polypropylene matrix based material and expressed in equation (6) as

$$
\mathrm{E}=2.222 \varphi^{3}+3.095 \varphi^{2}+3.368 \varphi+2.091
$$


Both the Halphin-Tsai and the Birintrup equations for elastic modulus of unidirectional fibre composites were confirmed to be appropriate for prediction of elastic modulus of nanofiller composites with polymer matrix.

\section{REFERENCES}

[1] Dieter, G.E. Engineering Design: A Material and Processing Approach, McGraw-Hill publishing, 3ed, pp.360-366, 2000.

[2] Zhang, Q.X., Z.Z., Yu, X.L., Xie and Mai, Y.W. Crystallization and Impact Energy of Polypropylene/CaCO3 Nanocomposites with Nonionic Modifier, Polymer, 45, pp.5985-5994.

[3] Hanim. H, Zarina, R.,Ahmad Fuad,M.Y.,Ishak.Z.A.M.,and Hassan, A . The Effect of Calcium carbonate Nano-filler on the mechanical properties and crystallization behaviour of Polypropylene, Malaysian Polymer Journal (MPJ), 2008, Vol 3, No. 12, pp. 38-49.

[4] Xie X.L, Q.X. Liu, R.K.Y.Li, X.P. Zhou, Q.X. Zhang, Z. Z. Yu and Y.Mai,(2004) "Rheological and Mechanical properties of $\mathrm{PVC} / \mathrm{CaCO}_{3}$ Nanocomposites prepared by In-situ polymerization, Polymer, 45, p.6665-6673.

[5] Di Lorenzo M. L, Enrico M. E, and Avell M. Thermal and morphological Characterization of Poly(ethylene terepthalate)/ Calcium Carbonate Nano composites, Journal of material service, 37, 2351-2358,2002.

[6] Chan C.M, Wu J, Li J.X, and Cheung Y.K. Polypropylene /Calcium Carbonate Nano composites, Polymer, 43, p.2981-2992, 2000.

[7] Eiras, D and Pessan, L.A. Crystallization behaviour of Polypropylene/Calcium carbonate nanocomposites, Technical paper presented on the $11^{\text {th }}$ International Conference on Advanced materials at Rio de Janeiro Brazil September pp.20-25, 2009.

[8] Guth E.J. Theory of Filler Reinforcement, Journal of Applied Physics, 1945, 16, pp.20

[9] Shigley, E.S and Mishchke, C. R. Mechanical Designers Work Book: Corrosion and Wear, McGraw-Hill Publishing, Tokyo, 1989, pp.16-40.

[10] Ihueze,C.C. and Mgbemena, C.O,. Effects of Reinforcement Combinations of Calcium carbonate nanofiller on the Mechanical and Creep properties of polypropylene (awaiting publication in JMMCE, Ref2220504), 2010.

[11] Mickell P. Groover, Fundamentals of Modern manufacturing Engineering, John Wiley and Sons Inc, USA, pp.182-184, 2007.

[12] Crawford, R.J. Plastics Engineering, 3ed, BUTTERWORTH, HEINMANN, Oxford, 1998, pp.18-21, 41-52,168-180, 1998.

[13] http//:en.wikipedia.org/wiki/yield. 